\title{
Advanced Gust Load Alleviation using Dynamic Control Allocation
}

\author{
Manuel Pusch*and Thiemo Kier ${ }^{\dagger}$ \\ Institute of System Dynamics and Control, German Aerospace Center (DLR), 82234 Weßling, Germany \\ Martin Tang ${ }^{\ddagger}$ and Johannes Dillinger ${ }^{\S}$ \\ Institute of Aeroelasticity, German Aerospace Center (DLR), 37073 Göttingen, Germany \\ Daniel Ossmann ${ }^{\text {II }}$ \\ Department of Mechanical, Automotive and Aeronautical Engineering, \\ Munich University of Applied Sciences (MUAS), 80335 Munich, Germany
}

\begin{abstract}
Active control techniques play a key role in today's aircraft developments to reduce structural loads during gust encounter and thereby enable highly fuel-efficient aircraft designs. The performance of such gust load alleviation (GLA) systems, however, is often limited by physical limitations in the actuation system. In this paper, a novel GLA control approach is presented which exploits actuator redundancies to avoid performance degradations when individual actuators are driven to their limits or become faulty. To that end, a baseline controller for damping loads-dominating aeroelastic modes is extended with a dynamic control allocation system for handling constrained actuators. Thereby, virtual control inputs, generated for a targeted aeroelastic mode control, are distributed to the actual control inputs in an optimal way. To do so, a convex optimization problem is formulated which is solved in real time with the goal to minimize performance degradations due to actuator constraints. The effectivity of the presented GLA controller is experimentally validated on a highly flexible wing in a wind tunnel considering different actuator constraints and gust excitations at multiple airspeeds.
\end{abstract}

\section{Introduction}

$\mathbf{T}^{\mathrm{N}}$ order to improve aircraft performance, structural weight reductions and aerodynamically efficient high aspect ratio Iwings play a key role [1]. This, however, typically leads to an increased flexibility of the aircraft structure with an increased sensitivity to gust encounters [2]. To counteract this adverse effect, active control technologies are becoming increasingly important allowing for considerable reductions of structural loads during gust encounter. Such gust load alleviation (GLA) systems aim at redistributing lift by a coordinated deflection of control surfaces. Thereby, physical limitations in the actuation system typically also limit achievable performance of the GLA system.

For the design of an according GLA controller, this means that actuator saturation needs to be explicitly considered. This can be achieved, e.g., by an extensive controller tuning based on nonlinear simulations with constrained actuator models, see, e.g., [3-5]. Another approach which is also used in industry is described in [6], where wind-up and stability issues are effectively avoided by setting thresholds for activating the GLA system. A similar, bang-bang like GLA controller is proposed in [7] which is only activated when the output of a specially developed disturbance estimator exceeds a certain threshold. Other approaches explicitly handling actuator constraints include model predictive control (MPC) theory as proposed in, e.g., [8-10]. However, MPC algorithms generally require simple simulation models for real-time capability, which makes an application to flexible aircraft difficult due to the generally complex aircraft dynamics [11]. In case of over-actuation, saturation effects may also be avoided by redistributing the demanded control power in real-time using dynamic control allocation [12]. For example, [13] implements a control allocation system for primary flight control which considers structural load constraints as a secondary objective. A similar approach is followed in [14], where the control allocation problem is formulated in terms of a quadratic program instead of a

\footnotetext{
*Research Fellow, Department of System Dynamics and Control, DLR, manuel.pusch@dlr.de

$\dagger$ Research Fellow, Department of System Dynamics and Control, DLR, thiemo.kier@dlr.de

$\dashv$ Research Fellow, Department of Structural Dynamics and System Identification, DLR, martin.tang@dlr.de

${ }_{\text {}}^{\S}$ Research Scientist, Department of Loads Analysis and Aeroelastic Design, DLR, johannes.dillinger@dlr.de

IIProfessor, Department of Mechanical, Automotive and Aeronautical Engineering, MUAS, daniel.ossmann@hm.edu, AIAA Senior Member
} 
linear program. Note that both dynamic control allocation and MPC do not only allow for effectively handling saturation effects but also actuator faults, see, e.g., $[15,16]$.

In this paper, a novel GLA system is presented which integrates an aeroelastic mode controller with a dynamic control allocation system. The aeroelastic mode controller aims at damping loads-dominating aeroelastic modes and is based on blending control inputs and measurement outputs. The blending of inputs and outputs reduces the original multiple-inputs and multiple-outputs (MIMO) control problem to a a single-input and single-output (SISO) one, which is considerably less complex and generally easier to tune. The structure of the blending-based modal controller further provides a convenient way to consider constrained actuators by extending it with a dynamic control allocation system. The dynamic control allocation system is only active in case of actuator saturation or faults and aims at maintaining controller performance by redistributing the demanded control power. If redistribution is insufficient, controller performance is degraded in systematic way, where weighting factors allow for prioritizing the individual aeroelastic mode controllers.

The general GLA control approach with its two modules - the aeroelastic mode controller and the dynamic control allocation - is described in Section II. To evaluate the effectivity of the proposed GLA control approach, it is applied in Section III to a highly flexible wing, where the controller is designed and tuned using a detailed simulation model of the wing. Eventually, the developed GLA controller is validated in an extensive wind tunnel test campaign, where a special focus is put on studying the effect of actuator constraints. The promising results of the wind tunnel test are discussed in Section IV.

\section{Gust Load Alleviation using Dynamic Control Allocation}

An advanced GLA system is presented which is combines a blending-based modal controller [17] with a novel dynamic control allocation for handling actuator constraints. In what follows both the blending-based modal control law and the dynamic control allocation are described and scheduling of the overall controller is discussed.

\section{A. Aeroelastic Mode Control using Blended Inputs and Outputs}

Assuming that the considered aeroelastic system is linear time-invariant (LTI) and diagonalizable, it can be described as

$$
G(s)=\sum_{i=1}^{n_{i}} M_{i}(s),
$$

where

$$
M_{i}(s)= \begin{cases}\frac{R_{i}}{s-p_{i}} & \text { if } \mathfrak{J}\left(p_{i}\right)=0 \\ \frac{R_{i}}{s-p_{i}}+\frac{\bar{R}_{i}}{s-\bar{p}_{i}} & \text { otherwise. }\end{cases}
$$

In Equations (1) and (2), $M_{i}(s)$ describes an aeroelastic mode whose dynamics are defined by either a single real pole $p_{i}$ with an imaginary part $\mathfrak{J}\left(p_{i}\right)=0$ or a conjugate complex pole pair $p_{i}$ and $\bar{p}_{i}$. Each pole $p_{i}$ is associated with a residue matrix $R_{i}$, where the residue matrices of a conjugate complex pole pair are also conjugate complex. Note $G(s)$ and $M_{i}(s)$ feature the same number of $n_{u}$ inputs and $n_{y}$ outputs. Furthermore, it is noted that in Equation (1), the direct feedthrough term is neglected since it is generally not present in mechanical systems.

The task of controlling a single aeroelastic mode $M_{j}(s) \in\left\{M_{m}(s)\right\}$ an aeroelastic system is challenging, especially when the number of control inputs or measurement outputs is large. To reduce the complexity of the control problem, it is proposed to weight and sum the measurement signals such that the resulting virtual measurement output $v_{y, j}$ "best" represents the response of the targeted aeroelastic mode $M_{j}(s)$. Similarly, it is proposed to generate a virtual control input $v_{u, j}$ which is distributed to available control inputs such that the targeted mode is "best" controlled. To generate the virtual inputs and outputs, the input and output blending vectors $k_{u, j} \mathbb{R} n_{u}$ and $k_{y, j} \in \mathbb{R} n_{y}$ are applied to the aeroelastic system $G(s)$. In doing so, the targeted mode can be controlled by a simple SISO controller $\lambda_{j}(s)$. Hence, the MIMO control design problem becomes a SISO one, where the resulting feedback controller is given as

$$
K_{j}(s)=k_{u, j} \lambda_{j}(s) k_{y, j}^{T}
$$


For computing input and output blending vectors $k_{u, j}$ and $k_{u, j}$ which allow for "best" controlling $M_{j}(s)$, different strategies have been proposed For example, [18] derive blending vectors which enable damping the targeted mode with a static feedback gain of minimum magnitude. This approach is extended in or [19], where the poles of the targeted mode can be moved to an arbitrary location in the complex plane. Another approach is to aim at a maximum controllability and observability of the targeted mode while decoupling it from the remaining system dynamics. Considering the $\mathcal{H}_{-}$ index as a joint controllability/observability measure, an according algorithm is derived in [20] and extended to linear parameter-varying (LPV) systems in [21]. Similarly, [22] uses the Generalized Kalman - Yakubovich - Popov Lemma for computing blending vectors based on the $\mathcal{H}_{-}$index. In [17], another approach is proposed which aims at maximizing the $\mathcal{H}_{2}$ norm of the blended mode. For a more detailed review on different blending methods see, e.g., [23]. Note that herein, the $\mathcal{H}_{2}$-optimal blending approach from [17] is used, see also Section III.B.

Considering multiple mods $M_{j}(s), j=1,2, . ., n_{j}$ to be controlled, it is proposed to superimpose the individual modal controllers $K_{j}(s)$ from Equation (3). The overall feedback controller is thus given as

$$
K(s)=K_{u} \Lambda(s) K_{y}^{T}=\sum_{j=1}^{n_{j}} K_{j}(s),
$$

where the input and output blending vectors of all modal controllers are collected as columns in the input and output blending matrices $K_{u}=\left[\begin{array}{llll}k_{u, 1} & \ldots & k_{u, n_{j}}\end{array}\right]$ and $K_{y}=\left[\begin{array}{llll}k_{y, 1} & \ldots & k_{y, n_{j}}\end{array}\right]$, and the respective SISO controllers are collected on the diagonal of

$$
\Lambda(s)=\left[\begin{array}{ccc}
\lambda_{1}(s) & \cdots & 0 \\
\vdots & \ddots & \vdots \\
0 & \cdots & \lambda_{n_{j}}(s)
\end{array}\right]
$$

The overall controller structure is depicted in Fig. 1. Note that to avoid any interaction of the individual modal controllers, they should be well decoupled. This needs to be considered during modal controller design as discussed in, e.g., [23].

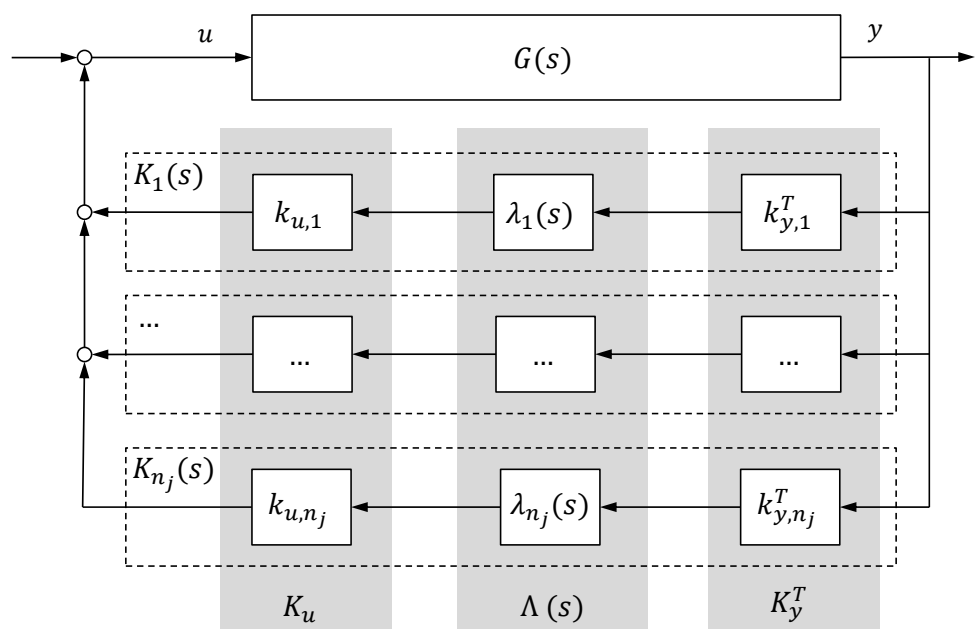

Fig. 1 Blending-based modal feedback controller where each $K_{j}(s)$ controls a mode $M_{j}(s)$.

\section{B. Dynamic Control Allocation for Handling Actuator Constraints}

In the case of actuator constraints like saturation or faults, controller performance may be degraded or, in the worst-case, the closed-loop may even become unstable. To counteract this, it is proposed to augment the existing blending-based controller with a dynamic control allocation. In general, the objective of a control allocation algorithm is to compute a control input $u$ from a virtual control command $v_{u}$ which can be realized by the given set of actuators [12]. Assuming sufficient control authority, the input blending matrix $K_{u}$ can hence be seen as a control allocation since it distributes $v_{u}$ to $u$. However, this kind of static control allocation may be unrewarding if control authority 
is limited, i.e., the capability of the actuators is constrained. In that case, it is recommended to use dynamic control allocation which also considers actuator constraints. Typically, the dynamic control allocation problem is formulated as an optimization problem which is solved at each time sample. If the optimization problem is infeasible, i.e., the virtual control command cannot be realized adequately, controller performance is necessarily degraded, which is commonly described by a so-called slack variable.

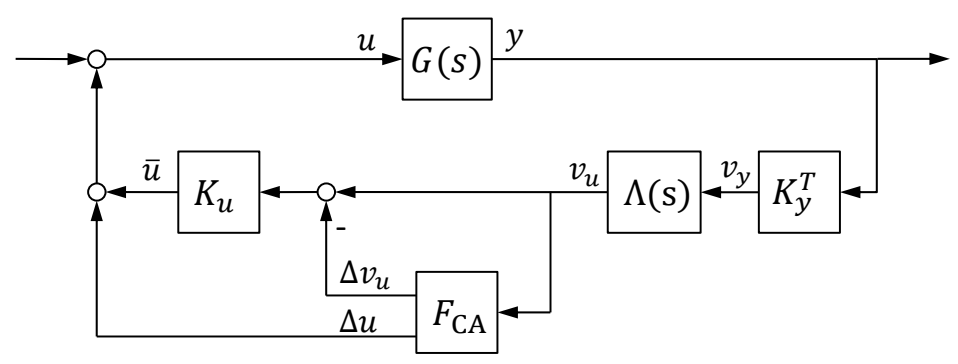

Fig. 2 Closed-loop interconnection of plant $G(s)$ with blending-based controller $K(s)=K_{u} \Lambda(s) K_{y}^{T}$ and dynamic control allocation extension $\boldsymbol{F}_{\mathrm{CA}}$.

The control allocation scheme proposed herein is depicted in Fig. 2, where the nominal blending-based feedback controller $K(s)$ is described in Equation (4). The given scheme is a reformulation of the more common control allocation scheme described, i.e., in [12] such that the augmentation of the blending-based nominal controller becomes clearly visible. In general, it is desired that the dynamic control allocation is only active when the nominal control input $\hat{u}=K_{u} v_{u}$ exceeds the upper or lower actuator limit $u_{\min } \in \mathbb{R}_{\leq 0}^{n_{u}}$ or $u_{\max } \in \mathbb{R}_{\geq 0}^{n_{u}}$. If this is the case, a control input redistribution vector $\Delta u \in \mathbb{R}^{n_{u}}$ is added to the nominal control input $\hat{u}$ such that the resulting control input is within actuator limits and nominal controller performance is not affected. In case no feasible $\Delta u$ can be found, controller performance is degraded such that a feasible $\Delta u$ exists, which is realized by reducing the virtual control command $v_{u}$ by

$$
\Delta v_{u}=\operatorname{diag}(\tau) v_{u}=\left[\begin{array}{ccc}
\tau_{1} & \ldots & 0 \\
\vdots & \ddots & \vdots \\
0 & \ldots & \tau_{n_{j}}
\end{array}\right] v_{u}
$$

In Equation (6), $\tau \in \mathbb{R}^{n_{j}}$ denotes the slack variable, where its elements $\tau_{j} \in\left[\begin{array}{ll}0 & 1\end{array}\right]$ indicate the relative performance degradation of the individual SISO controllers $c_{j}, j=1, \ldots, n_{j}$. This means that for $\tau_{j}=1$, the respective SISO controller $c_{j}$ is actually inactive and $\tau_{j}=0$ denotes the nominal case without any performance degradation.

Eventually, the resulting control input is computed as

$$
u=K_{u} v_{u}-K_{u} \operatorname{diag}(\tau) v_{u}+\Delta u=\underbrace{\hat{u}-K_{u} \Delta v_{u}}_{\bar{u}}+\Delta u,
$$

where $\bar{u}$ denotes the reduced nominal control input. All in all, the described control allocation scheme features three different modes which are summarized in Table 1.

Table 1 Control allocation modes.

\begin{tabular}{lccl}
\hline mode description & $\Delta u$ & $\tau$ & control input \\
\hline unconstrained (nominal control) & $=0$ & $=0$ & $u_{\min } \leq \hat{u} \leq u_{\max }$ \\
redistribution only & $\neq 0$ & $=0$ & $u_{\min } \leq \hat{u}+\Delta u \leq u_{\max }$ \\
redistribution + performance reduction & $\neq 0$ & $\neq 0$ & $u_{\min } \leq \bar{u}+\Delta u \leq u_{\max }$ \\
\hline
\end{tabular}

For computing a suitable control input redistribution $\Delta u$, the objective is that the redistribution vector should be minimal in terms of some $p$-norm, where $p$ is typically chosen as 1 or 2 . To maintain controller performance, $\Delta u$ is enforced to be orthogonal on the input blending matrix $K_{u}$ and hence on the nominal control input $\hat{u}=K_{u} v_{u}$. In case it 
is further desired that certain residual dynamics captured in $G_{r}(s)=C_{r}\left(s I-A_{r}\right)^{-1} B_{r}$ are not excited, it proposed to enforce that $\Delta u$ is orthogonal on $B_{r}$, i.e., $B_{r} \Delta u=0$. Additionally, it is also desired that the performance degradation is minimal, which is considered by further minimizing the $p$-norm of the slack variable $\tau$. Eventually, the optimization problem for dynamic control allocation is formulated as

$$
\begin{array}{ll}
\underset{\Delta u \in \mathbb{R}^{n_{u}}, \tau \in \mathbb{R}^{n_{j}}}{\operatorname{minimize}} & \left\|W_{u} \Delta u\right\|_{p}+\left\|W_{\tau} \tau\right\|_{p} \\
\text { subject to } & 0 \leq \tau \leq 1 \\
& u_{\min } \leq u \leq u_{\max } \\
& \Delta u^{T}\left[\begin{array}{ll}
K_{u} & B_{r}^{T}
\end{array}\right]=0,
\end{array}
$$

where $u$ is computed according to Equation (7). In Equation (8), the positive definite weighting matrices $W_{u} \in \mathbb{R}^{n_{u} \times n_{u}}$ and $W_{\tau} \in \mathbb{R}^{n_{j} \times n_{j}}$ are introduced in the objective function for balancing the priority of the individual control inputs and modal SISO controllers. Typically, both matrices are diagonal and $W_{\tau}$ is chosen much larger than $W_{u}$ in order to prioritize redistribution over performance reduction. Besides, the constraint $0 \leq \tau \leq 1$ is considered in Equation (8) to ensure that controller performance is only decreased but not increased $(\tau>0)$ or even "inverted" $(\tau<0)$. Note that to ensure a feasible solution, it is required that $u_{\min } \leq 0$ and $u_{\max } \geq 0$, because in that case a feasible solution can always be obtained by turning off the controller setting $\tau=1$ and $\Delta u=0$. To further consider actuator faults, the actuator constraints $u_{\min } \leq u \leq u_{\max }$ are commonly adapted in real-time [12], e.g., by setting the upper and lower limits of a faulty actuator to zero. This ensures that the faulty actuator is not used and the desired control commands are redistributed. Furthermore, it is noted that Equation (8) can be reformulated to consider also rate limitations of the actuation system, see, e.g.Johansen2013 for more details.

In case $p=1$, Equation (8) represents a linear program which can be solved efficiently by using, e.g., the simplex method [24]. In case $p=2$, a quadratic program is obtained which is convex and hence can be solved efficiently by using, i.e., interior-point methods [25]. In general, the 2-norm minimization tends to use a large number of actuators to a smaller degree in comparison to a 1-norm minimization, which commonly favors the use of a smaller number of actuators but to a higher degree [26]. Furthermore, it is noted that uniqueness of a feasible solution is usually only guaranteed when formulating the objective function in terms of the 2-norm [12]. In either case, it is recommended to eliminate the constraint $\Delta u^{T}\left[\begin{array}{ll}K_{u} & B_{r}^{T}\end{array}\right]=0$ in Equation (8) and directly search for a $\Delta u$ in the left null space of $\left[\begin{array}{ll}K_{u} & B_{r}^{T}\end{array}\right]$. This allows for greatly reducing computational effort by reducing the number of optimization variables. See [23] for more details.

\section{Controller Scheduling for Handling Parameter Variations}

In order to handle parameter-varying system dynamics, gain scheduling techniques may be applied [27]. The most straight-forward approach thereby is to design separate LTI controllers at different operation points using linearized models and interpolate in between them. Another, mathematically more profound framework is LPV control [28-30], where models of reduced complexity are generally required for numerical reasons [31]. In other words, LPV control quickly comes to its limits in case of a large number of inputs, outputs, states, or varying parameters. A similar challenge is faced in parametric structured control design [32,33], where numerical limits are generally imposed by the complexity of the chosen controller structure. This includes, amongst others, the bad scalability with the number of inputs and outputs which is directly connected with the number of free tuning parameters to be optimized.

To overcome this issue, the modal control approach presented in Section II.A provides new possibilities to handle parameter variations. This is achieved by splitting the design procedure into a blending vector design and a SISO controller design. In this way, each part can be scheduled separately leading to less complex design problems which are numerically better tractable. Depending on the dynamics of the underlying parameter-varying system, it may even be sufficient to schedule only one part of the modular controller while keeping the rest constant. For instance, in many aeroelastic applications, the shape of the targeted modes change only marginally within the operation range while their frequency and gain vary considerably. In this case, it is proposed to keep the blending vectors constant and only schedule the dynamics of the SISO controller, see [34] and Section III for examples. Furthermore, it is noted that in the proposed control approach, each aeroelastic mode is controlled by a separate modal controller, which gives the freedom to choose individual scheduling strategies for each targeted mode. Besides scheduling the aeroelastic mode controller, also the dynamic control allocation introduced in Section III.C may be scheduled. To that end, the involved matrices and actuator limits are parametrized, but considered constant when solving optimization problem (8) at each individual time sample. 


\section{Application to Experimental Flexible Wing}

The effectiveness of the GLA system described in Section II is demonstrated on an experimental flexible wing which is depicted in Fig. 3. The experimental wing is designed within the DLR project KonTeKst (Configurations and Technologies for Short Range Aircraft) [35]. To reduce the structural loads of the flexible wing during vertical gust encounters, simulated by pitching the wing, a GLA controller is designed with the goal to reduce the wing root bending moment (WRBM). As described in Section II, the GLA system consists of two modules: an aeroelastic mode controller and a dynamic control allocation system. In the sections below, the two controller modules are derived and validated in simulations using a detailed simulation model of the flexible wing. The results of the experimental controller validation are presented in Section IV.

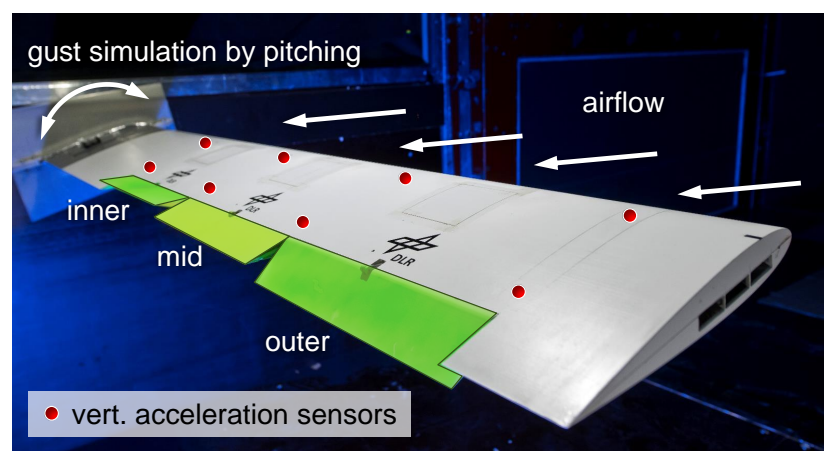

Fig. 3 Flexible wing mounted in wind tunnel.

\section{A. Experimental Flexible Wing}

The experimental wing is $1.6 \mathrm{~m}$ long and features a symmetric NACA 0015 profile with a chord length of $0.25 \mathrm{~m}$. It consists of glass fiber skins and a foam core, making it highly flexible and sensitive to gust excitations. The dynamics of the highly flexible wing are captured in an aeroelastic model which couples forces from aerodynamics and structural dynamics [36]. The forces from structural dynamics are derived in modal space using the finite element method [37]. The aerodynamic forces are modeled in the frequency domain using the doublet lattice method and transformed into time domain by rational function approximation.

For controlling the flexible wing, it is equipped with $n_{y}=8$ vertical acceleration sensors and $n_{u}=3$ trailing edge flaps, see Fig. 3. The transfer functions of the flap actuators are identified in a separate actuator testbed and added to the aeroelastic simulation model [36, 38]. Furthermore, structural loads are measured at the wing root using a piezoelectric balance, which is used for evaluating controller performance and also included in the model. The overall system dynamics are integrated in a single LPV model, considering the free stream velocity as a varying parameter. For more details on modeling the flexible wing see [36-38].

\section{B. Aeroelastic Mode Controller Design using $\mathcal{H}_{2}$-optimal Blending}

The goal of the GLA system is to reduce the WRBM by actively damping the dominating aeroelastic modes. To achieve that, the airspeed of the aeroelastic model derived in Section III.A is fixed at different values and a modal decomposition is carried out on the resulting LTI systems.

Thereby, it becomes clear that the lightly damped first and second wing bending modes dominate the WRBM. This can also be seen in Fig. 4(a), where the two peaks around $8 \mathrm{~Hz}$ and $46 \mathrm{~Hz}$ are associated to the first and second wing bending mode, respectively. The second wing bending mode, however, is marginally excited during gust encounters due to its high natural frequency and the low-pass characteristic of gusts. Thus, the objective for blending-based GLA controller design is to increase the damping of the first wing bending mode.

For the blending vector design, the $\mathcal{H}_{2}$-optimal blending approach from [39] is applied. The approach aims at maximizing the joint controllability and observability of the targeted mode $M(s)$ with blended inputs and outputs in terms of the $\mathcal{H}_{2}$ norm. More precisely, the goal in blending vector design is to maximize $\left\|k_{y}^{T} M(s) k_{u}\right\|_{\mathcal{H}_{2}}$, where the length of the input and output blending vectors $k_{u}$ and $k_{y}$ is restricted to one, respectively. This allows for actively damping the blended target mode with a SISO controller of minimum gain. 

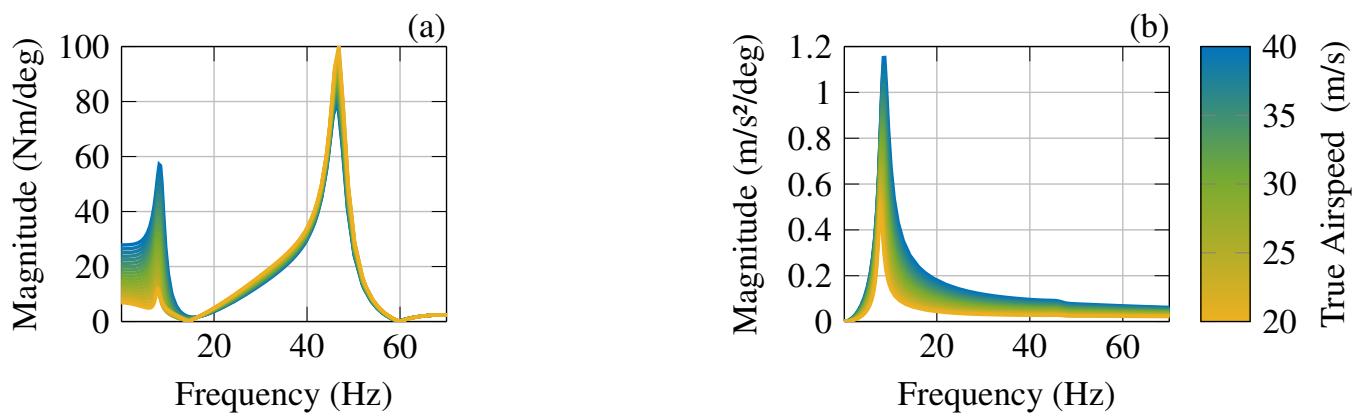

Fig. 4 Selected frequency responses at different airspeeds: (a) from pitch angle (deg) to WRBM (Nm), (b) from blended inputs (deg) to blended outputs $\left(\mathrm{m} / \mathrm{s}^{2}\right)$.

As control inputs, the three flap deflection commands are considered and as measurement outputs, all eight vertical acceleration sensor signals are considered. Since the shape of the first wing bending mode varies only slightly within the considered airspeed range, the corresponding input and output blending vectors $k_{u}$ and $k_{y}$ are computed at a single airspeed $v_{\text {tas }}=40 \mathrm{~m} / \mathrm{s}$ and held constant for all airspeeds. Applying the computed blending vectors isolates the first wing bending mode as depicted in Fig. 4(b).

As SISO controller, a gain-scheduled proportional-integral controller is chosen which is augmented with the band-pass filter $W_{\mathrm{bp}}$. The band-pass filter restricts controller activity to the frequency range of the first wing bending mode and suppresses noise at high frequencies. The proportional and integral gains, $k_{p}\left(v_{\text {tas }}(t)\right)$ and $k_{i}\left(v_{\text {tas }}(t)\right)$, are scheduled with true airspeed $v_{\text {tas }}(t)$. Both gains are manually tuned at a true airspeed of $20 \mathrm{~m} / \mathrm{s}, 30 \mathrm{~m} / \mathrm{s}$ and $40 \mathrm{~m} / \mathrm{s}$, and linearly interpolated in between. The controller is tuned in such a way that the damping of the first wing bending mode is maximized considering flap deflection limits and robustness requirements. The maximum flap deflections are given as $\pm 10^{\circ}$ and are evaluated in terms of closed-loop simulations carried out for the worst-case harmonic pitch excitation causing $\pm 10 \mathrm{~cm}$ wing tip deflection. The robustness of the control loop is evaluated in terms of the symmetric disk margin [40], which is computed at each of the multiple inputs and multiple outputs (loop-at-a-time). As a minimum requirement, a $45^{\circ}$ phase margin is demanded, which is equivalent to a $7.7 \mathrm{~dB}$ gain margin. Eventually, the nominal GLA controller computes the flap deflection commands as

$$
u(t)=k_{u} W_{\mathrm{bp}}\left(k_{p}\left(v_{\mathrm{tas}}(t)\right) k_{y}^{T} y(t)+\int_{0}^{t} k_{i}\left(v_{\text {tas }}(\tau)\right) k_{y}^{T} y(\tau) \mathrm{d} \tau\right),
$$

where $y$ collects the eight vertical acceleration measurements. Furthermore, it is noted that the true airspeed $v_{\text {tas }}$ is separately measured by a pitot tube mounted in the windtunnel.

The achieved damping of the first wing bending mode for fixed airspeeds is depicted in Fig. 5. It can be seen that the relative damping is increased by a factor of more than five at higher airspeeds while it is not even tripled at lower airspeeds. This is due to the fact that flap efficiency increases with increasing airspeed. Furthermore, it is noted that the poles of the remaining aeroelastic modes are hardly affected when closing the loop. This is a result of the band-pass behavior of the SISO controller and the isolation of the first wing bending mode via input and output blending.

\section{Control Allocation Design}

In case of actuator saturation or faults, the performance of the derived aeroelastic mode controller for GLA is generally decreased. Since the considered flexible wing is actually over-actuated, this performance loss can be effectively avoided or at least reduced by means of dynamic control allocation. Implementing the dynamic control allocation proposed in Section II.B, the actual control inputs $u$ are computed from the virtual control input $v_{u}$ such that nominal controller performance is maintained as good as possible in presence of actuator constraints. The corresponding optimization problem is given in Equation (8), where $p=2$ is chosen here. This means that the objective is to minimize the 2-norm of the slack variable $\tau$ and redistribution vector $\Delta u$, which are weighted by $W_{\tau}=2 \times 10^{9}$ and $W_{u}=I$, respectively. In doing so, it is enforced that control input redistribution is preferred over controller performance degradation, where $W_{\tau}$ is chosen as large as possible without causing numerical issues. Furthermore, it is noted that no decoupling constraints are considered, i.e., $B_{r}$ is neglected in Equation (8). To solve the optimization problem (8), which is a convex quadratic program, numerous computationally efficient methods are readily available. Herein, a derivative of the KWIK algorithm described in [41] is used, where the lower and upper actuator limits $u_{\min }$ and $u_{\max }$ can be updated in real-time. This allows, e.g., to consider a faulty actuator by setting both of its limits to zero. 


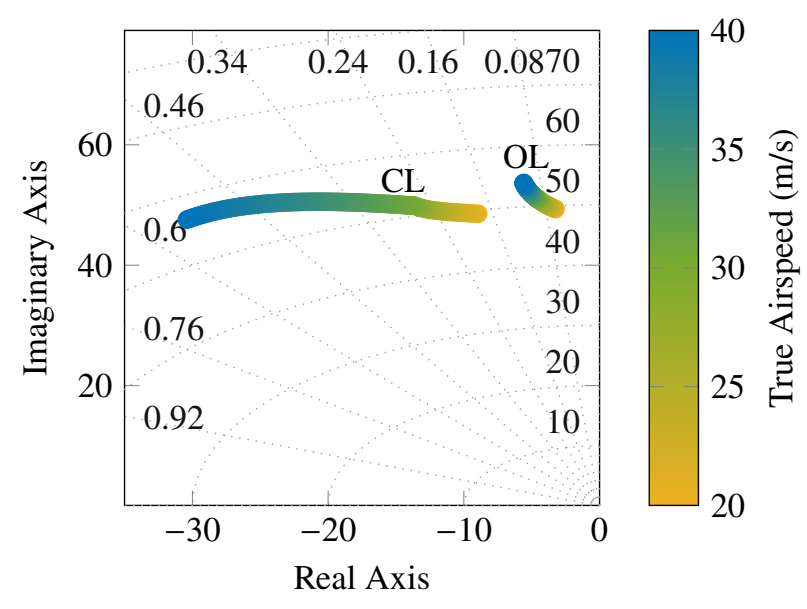

Fig. 5 Comparison of the open-loop (OL) and closed-loop (CL) poles of the first wing bending mode at different airspeeds.

\section{Controller Validation in Wind Tunnel Experiments}

The GLA system derived in Section III is experimentally validated at the Crosswind Simulation Facility of the German Aerospace Center (DLR) in Göttingen. The maximum flow velocity of the closed-circuit wind tunnel is $65 \mathrm{~m} / \mathrm{s}$ and the dimension of the test section is $2.4 \mathrm{~m}$ (width) by $1.6 \mathrm{~m}$ (height). The flexible wing mounted in the wind tunnel is depicted in Fig. 3. At the wing root the wing is attached to the pitch excitation system, which is installed outside of the wind tunnel such that only the wing is exposed to the airflow. Just as the control system, which runs on a Jäger ADwin Gold real-time system at a sampling rate of $1 \mathrm{kHz}$. The validation of the GLA system is performed at different airspeeds between $20 \mathrm{~m} / \mathrm{s}$ and $40 \mathrm{~m} / \mathrm{s}$. The following result discussion primarily focuses on an airspeed of $40 \mathrm{~m} / \mathrm{s}$, where it is noted that similar results are obtained at all airspeeds.

\section{A. Aeroelastic Mode Controller Validation}

The performance of the aeroelastic mode controller, i.e., the GLA system without dynamic control allocation, is evaluated by performing open- and closed-loop experiments for different gust excitations. The gust excitations are simulated by pitching the wing and include both discrete and stochastic gusts. Additionally, sweep excitations are carried out. Each pitch excitation is performed with and without the GLA controller at $20 \mathrm{~m} / \mathrm{s}, 30 \mathrm{~m} / \mathrm{s}$ and $40 \mathrm{~m} / \mathrm{s}$ true airspeed, where the resulting WRBMs are compared for controller performance evaluation. Note that in this section, only results are given where actuator constraints are not active. The effect of actuator constraints on the performance of the aeroelastic mode controller is discussed within Section IV.B.

For discrete gusts, the "1-cos" gust model from [42] is considered. To identify the critical "1-cos" gust, first the gust length $H$ is determined which causes the largest WRBM for a moderate design speed. As expected, gust lengths yielding gusts in the frequency range of the first wing bending mode cause the largest WRBM. Then, the design speed $v_{\mathrm{ds}}$ is adjusted such that the WRBM does not exceed $100 \mathrm{~N} \mathrm{~m}$. In Fig. 6, the input and output data for the critical "1-cos" gust excitation at $40 \mathrm{~m} / \mathrm{s}$ true airspeed is shown. The pitch excitation signal for both experiments open- and closed-loop is depicted in Fig. 6(a) together with the closed-loop flap deflection commands. Comparing the inner, mid, and outerflap deflections, it can be seen that the ratio between them remains constant as a result of input blending. Thereby, the outer flap is deflected the most and the inner flap the least, which reflects the shape of the first wing bending mode. The resulting reduction of the WRBM is visible in Fig. 6(b), where the open- and closed-loop measurements are compared. Clearly, both the peak and the settling time of the WRBM are reduced when closing the loop confirming the load reduction capabilities of the aeroelastic mode controller. Note that the WRBM measurement is only used for controller performance analysis and is not used in the feedback channels of the controller. Thereby it provides an independent variable to validate the performance of the developed algorithms. In Fig. 6(c) and 6(d), the measured data is further analyzed using fast Fourier transformations (FFTs). Fig. 6(c) illustrates that the "1-cos" pitch signal excites the system up to a frequency of about $15 \mathrm{~Hz}$, where the main energy is concentrated in the lower frequency range. In Fig. 6(d), the peak in the open-loop response lies at around $8 \mathrm{~Hz}$, which is caused by the lightly damped first wing bending mode. The aeroelastic mode controller, designed to increase the damping of this mode, flattens the peak and greatly reduces the output energy around its natural frequency, which is marked with a dotted line. 

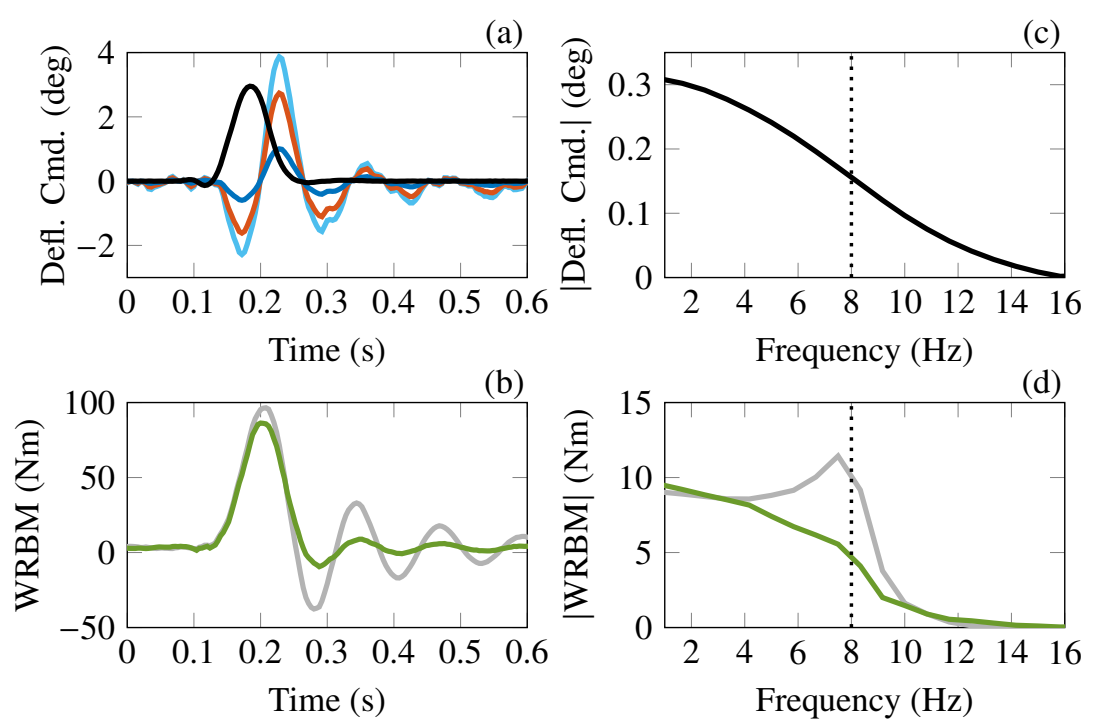

Fig. 6 Input and output data for the critical "1-cos" pitch excitation at $40 \mathrm{~m} / \mathrm{s}$ in the time domain in (a) and (b), and in the frequency domain in (c) and (d). In (a) and (c), the pitch excitation signal ( - ) is depicted. In (b) and (d), the open-loop (-) and closed-loop (-) wing root bending moment (WRBM) is compared. Additionally, the closed-loop inner $(-)$, mid $(-)$, and outer $(-)$ flap deflection commands are shown in (a).

To simulate stochastic gusts and test a broader frequency range, noise is commanded to the pitch motor. The noise is generated with a variance of $0.7 \mathrm{deg}$ within a frequency range of $3 \mathrm{~Hz}$ and $20 \mathrm{~Hz}$. A short section of the $300 \mathrm{~s}$ long excitation signal in time domain is depicted in Fig. 7(a). In Fig. 7(c), the FFT of the $300 \mathrm{~s}$ long input signal is depicted. Note that the signal has been smoothed for better readability. Clearly, the main energy of the input signal is concentrated in the lower frequency range between around $3 \mathrm{~Hz}$ and $10 \mathrm{~Hz}$. The reduction of the WRBM peaks by the controller are well visible by comparing the open-loop and closed-loop time signals in Fig. 7(b). FFTs of these two signals further reveal that energy around $8 \mathrm{~Hz}$ is clearly reduced in the closed-loop compared to the open-loop, see Fig. 7(d). Similarly to the "1-cos" excitation, the main energy in the output signal is concentrated around $8 \mathrm{~Hz}$ in the open-loop although this is not the case for the input signal. This is due to the lightly damped first bending mode at $8 \mathrm{~Hz}$. Increasing its damping with the aeroelastic mode controller leads to the desired reduction of energy in the output signal.

Finally, closed-loop sweep excitations are performed with the pitch motor at different airspeeds. The identified transfer functions are compared with the open-loop results from model validation in Fig. 8. The WRBM reduction resulting from actively damping the first wing bending mode is clearly visible at all airspeeds. Furthermore, the actual increase in modal damping is identified online and verified offline, where the corresponding values are given in Table 2. The relative damping of the first wing bending mode is increased by a factor of up to three. However, compared to the simulation results, the achieved damping is considerably smaller than expected, independent of the airspeed. The main reason for this lies in the large backlash of the flap actuation system, which is also discussed in the next subsection.

\section{B. Dynamic Control Allocation Validation}

Eventually, the aeroelastic mode controller augmented with dynamic control allocation is validated at different airspeeds and the representative results at $40 \mathrm{~m} / \mathrm{s}$ are discussed in detail. To that end, artificial actuator constraints are introduced in order to explicitly trigger the control allocation system. Note that actual actuator limits are much higher and never activated to prevent physical damage. Furthermore, this allows for comparisons with idealistic actuators which are not constrained, see also Section IV.B.

In a first step, closed-loop experiments are carried out for an $8 \mathrm{~Hz}$ sinusoidal pitch excitation of $1^{\circ}$ amplitude where flap deflection commands are artificially restricted to $\pm 1.5^{\circ}$. To evaluate the effect of the dynamic control allocation, the experiments are performed twice, with and without dynamic control allocation, where each experiment is carried out for $60 \mathrm{~s}$. Also taking into account the nominal closed-loop without any command limitations and only the aeroelastic 

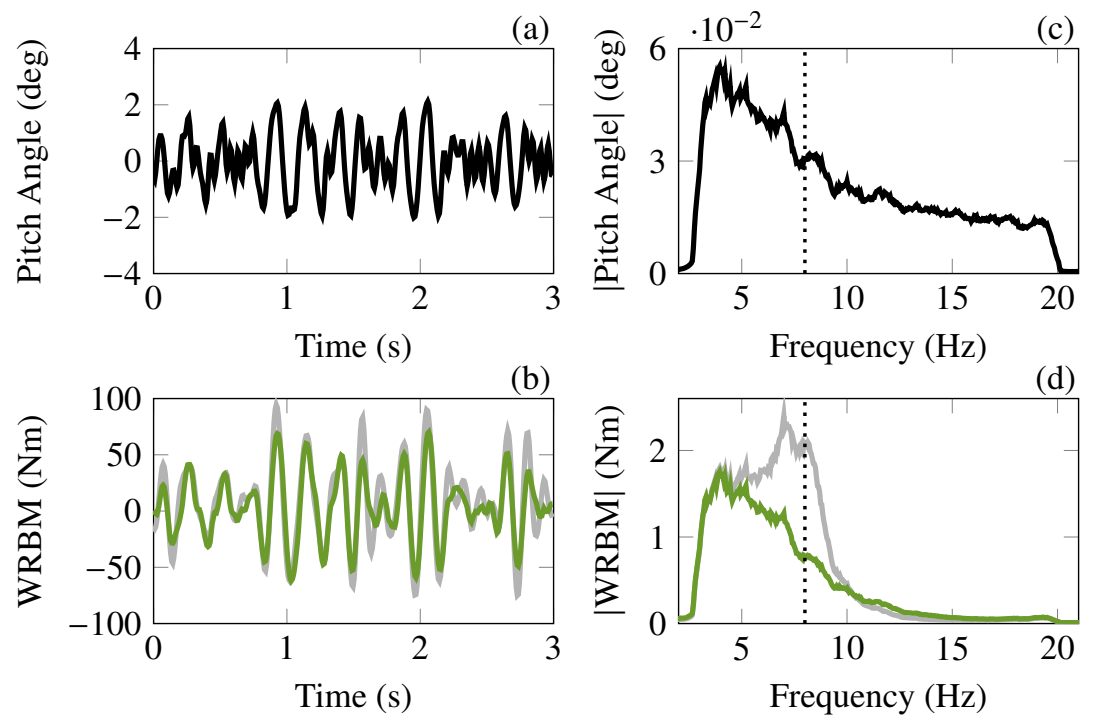

Fig. 7 Snippet of input and output data for noise excitation at $40 \mathrm{~m} / \mathrm{s}$ true airspeed in time domain in (a,b) and frequency domain in $(\mathbf{c}, \mathbf{d})$. In $(\mathbf{a , c})$ the pitch motor noise excitation $(-)$ is depicted, while in $(b, d)$ the open-loop $(-)$ and closed-loop $(-)$ wing root bending moment (WRBM) is compared.

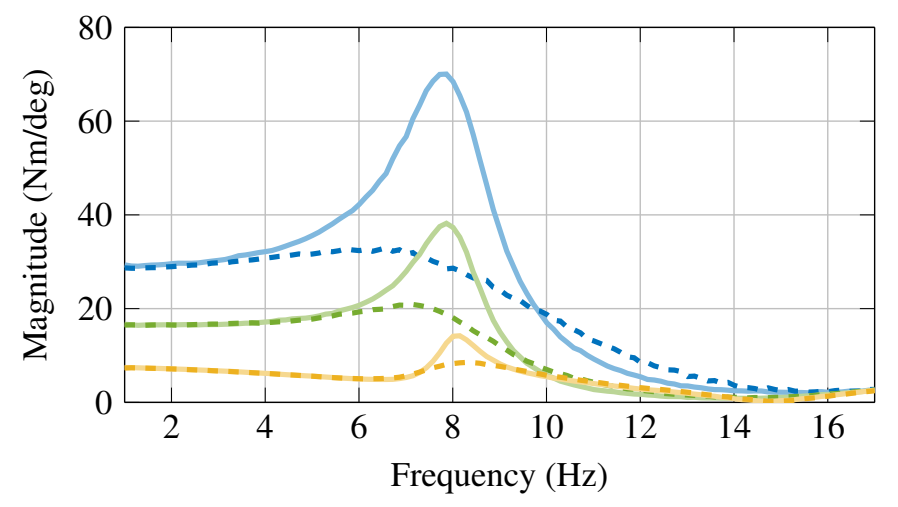

Fig. 8 Identified open-loop (solid lines) and closed-loop (dashed lines) frequency response from pitch angle (deg) to wing root bending moment $(\mathrm{Nm})$ at $\left.20 \mathrm{~m} / \mathrm{s}(-,--), 30 \mathrm{~m} / \mathrm{s}(-,--)^{-}\right)$and $40 \mathrm{~m} / \mathrm{s}\left(-,--^{--}\right)$true airspeed .

Table 2 Natural frequency and relative damping of the first bending mode in simulation (SIM) and experiment (EXP) for open- and closed-loop.

\begin{tabular}{ccccc}
\hline & \multicolumn{2}{c}{ natural frequency $(\mathrm{Hz})$} & \multicolumn{2}{c}{ relative damping (\%) } \\
\hline & open-loop & closed-loop & open-loop & closed-loop \\
true airspeed $(\mathrm{m} / \mathrm{s})$ & SIM / EXP & SIM / EXP & SIM / EXP & SIM / EXP \\
\hline 20 & $7.9 / 8.1$ & $7.9 / 8.2$ & $6.6 / 4.9$ & $18.1 / 11.0$ \\
30 & $8.2 / 8.0$ & $8.2 / 7.8$ & $8.9 / 8.4$ & $26.2 / 21.1$ \\
40 & $8.6 / 8.0$ & $9.0 / 8.1$ & $10.4 / 11.0$ & $53.8 / 34.4$ \\
\hline
\end{tabular}


Table 3 Controller configurations for dynamic control allocation validation.

\begin{tabular}{cccc}
\hline $\begin{array}{c}\text { Configuration } \\
\text { Name }\end{array}$ & $\begin{array}{c}\text { Associated } \\
\text { Color }\end{array}$ & $\begin{array}{c}\text { Command } \\
\text { Limitation }\end{array}$ & $\begin{array}{c}\text { Control } \\
\text { Allocation }\end{array}$ \\
\hline nominal & & off & off \\
restricted & & on & off \\
reconfigured & $\square$ & on & on \\
\hline
\end{tabular}

mode controller, three different controller configurations are tested in total, which are summarized in Table 3. For each of the three controller configurations, the resulting inner ( $\ldots .$.$) , mid (----), and outer (-)$ ) flap deflection commands are depicted in diagrams (a)-(c) of Fig. 9, respectively. Considering the nominal closed-loop, the flap deflections are not limited and no saturation occurs as depicted in Fig. 9(a). In comparison to that, control commands are limited to $\pm 1.5^{\circ}$ in the restricted controller configuration, which drives the outer and mid flap into saturation as depicted in Fig. 9(b). Thereby, the commands are generally increased since wing damping is degraded and higher accelerations are fed back to the flap actuators, as it is especially visible for the inner flap. Activating dynamic control allocation in the reconfigured case, the saturation of the mid and outer flap is compensated by a larger deflection of the non-saturated inner flap as shown in Fig. 9(c). In doing so, however, also the inner flap is driven into saturation. As soon as all three flap commands are saturated, nominal controller performance cannot be fully recovered anymore, which is indicated by the slack variable $\tau$ depicted in Fig. 9(d). Recall that the slack variable is considered as a direct measure for modal controller performance degradation, where $\tau=0$ means full controller performance and $\tau=1$ means that the controller is actually turned off.

(a)
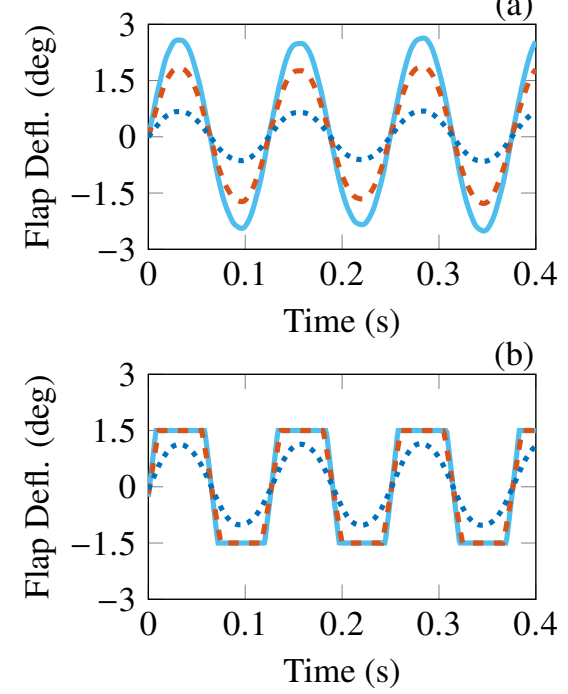

(c)
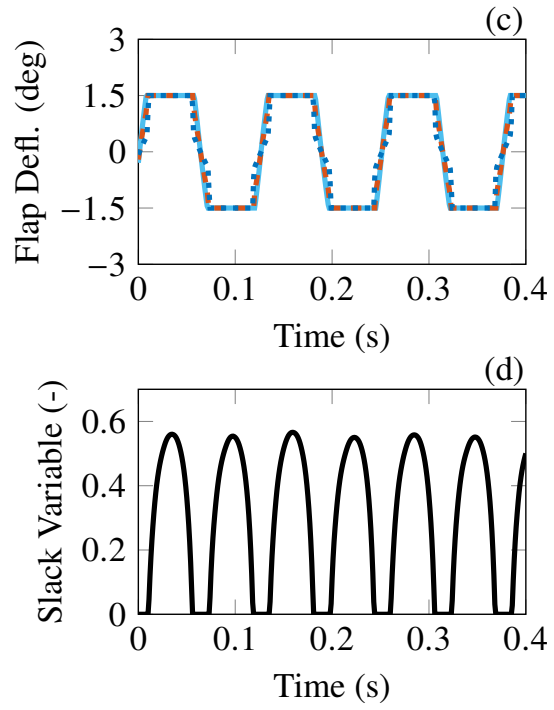

Fig. 9 Inner ( ....), mid (---), and outer (_- ) flap deflection commands for the "nominal" (a), "restricted" (b) and "reconfigured" (c) controller configuration. The slack variable $(-)$ of the "reconfigured" configuration with active dynamic control allocation is depicted in (d). The experiments are performed at $40 \mathrm{~m} / \mathrm{s}$ true airspeed with an $8 \mathrm{~Hz}$ sinusoidal pitch excitation of $1^{\circ}$ amplitude.

The performance of each controller configuration is validated in terms of the achieved WRBM reduction, which is computed as

$$
\eta_{\mathrm{f}}=1-\frac{\mathrm{f}\left(\left|y_{\text {wrbm,cl }}\right|\right)}{\mathrm{f}\left(\left|y_{\text {wrbm,ol }}\right|\right)},
$$

where $y_{\mathrm{wrbm}, \mathrm{ol}}$ and $y_{\mathrm{wrbm}, \mathrm{cl}}$ denote the open- and closed-loop WRBM measurements, and the function $\mathrm{f}=\{\max , \mathrm{rms}\}$ returns the maximum (max) or root-mean-square (rms) value of the given signal. In Fig. 10, $\eta_{\mathrm{rms}}$ and $\eta_{\text {max }}$ are depicted 
for the three different controller configurations given in Table 3. As expected, limiting flap deflections degrades nominal controller performance, where both $\eta_{\mathrm{rms}}$ and $\eta_{\max }$ show similar tendencies. Activating dynamic control allocation in the reconfigured controller configuration notably increases controller performance. However, nominal controller performance cannot be fully recovered since all flaps are driven into saturation.
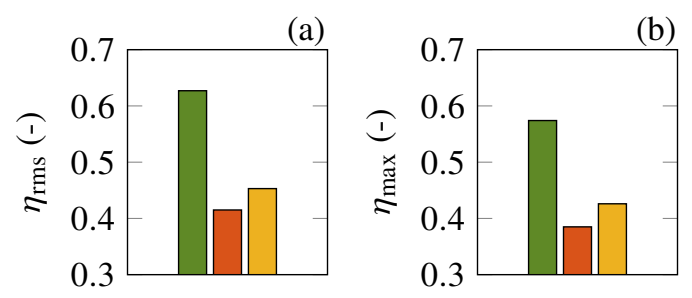

Fig. 10 Controller performance $\eta_{\mathrm{rms}}$ in (a) and $\eta_{\max }$ in (b) for the nominal ( $\square$ ), restricted ( $\square$ ), and reconfigured ( $\square$ ) controller configuration considering a $\pm 1.5^{\circ}$ command limitation for the latter two configurations. The experiments are performed at $40 \mathrm{~m} / \mathrm{s}$ true airspeed with an $8 \mathrm{~Hz}$ sinusoidal pitch excitation of $1^{\circ}$ amplitude.

In a second step, the capability of the dynamic control allocation to compensate actuator faults is investigated. To that end, a faulty outer flap actuator is simulated by freezing it at $0^{\circ}$. To deal with the faulty actuator, its lower and upper deflection limits are set to zero in the dynamic control allocation. As a consequence, it is enforced that the virtual control command is distributed to the non-faulty actuators while zero is commanded to the faulty one. For a comparative performance assessment, closed-loop experiments are carried out for each of the three controller configurations given in Table 3. But instead of limiting all flap deflections to $\pm 1.5^{\circ}$ in the restricted and reconfigured controller configuration, only the outer flap is limited here by freezing it at $0^{\circ}$. As gust excitations, the " $1-\cos "$ and noise excitation from the evaluation of the aeroelastic mode controller are taken into account besides the sinusoidal excitation from the previous experiments. The resulting WRBM reduction is again evaluated in terms of $\eta_{\mathrm{rms}}$ and $\eta_{\max }$, which are computed according to Equation (10) and compared in Fig. 11. As expected, controller performance is clearly reduced during fault scenarios where the outer flap is not working, compared to the nominal case, where all three flaps are operating regularly. Activating dynamic control allocation, nominal controller performance is recovered in the reconfigured case by compensating the faulty outer flap with larger deflections of the two remaining flaps. Taking a closer look on Fig. 11, however, it can be recognized that the WRBM reduction in the reconfigured case, with only two flaps in operation, is even slightly higher than the one resulting from the nominal case, with all three flaps working. After some closer investigation, this curious result is traced back to the large backlash of the inner flap, which is around $1.5^{\circ}$. Nonlinear simulations reveal that in the nominal case without any actuator fault, the inner flap is actually not, or only marginally deflected since the commanded deflections are mostly within free play. Obviously, this reduces nominal controller performance. In contrast, the reconfigured controller configuration commands larger deflections to the inner flap yielding a smaller performance loss due to the increased operation outside of the backlash area.
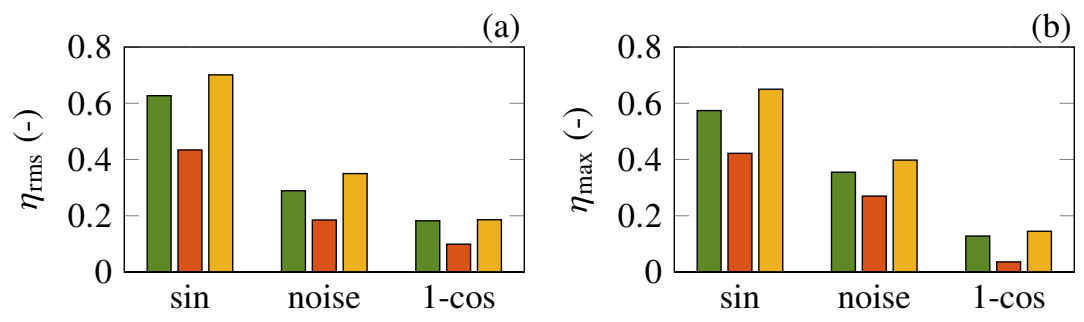

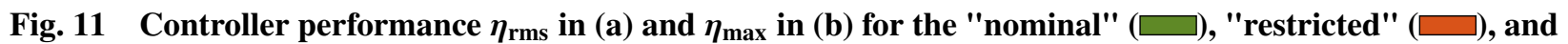
"reconfigured" ( $\square$ ) controller configuration considering a faulty outer flap for the latter two configurations. The experiments are performed at $40 \mathrm{~m} / \mathrm{s}$ true airspeed with sine, noise and "1-cos" pitch excitations.

In a final step, an entire fault scenario is tested where the outermost flap actuator is frozen at $2^{\circ}$ not from the beginning on but in the middle of the experiment. As excitation, a sinusoidal pitching motion around the frequency of the first bending mode at $8 \mathrm{~Hz}$ and $1^{\circ}$ amplitude is chosen. The resulting control and measurement signals are depicted in Fig. 12 and can be divided into four main segments. The test is started in open-loop, i.e., only the excitation signal at 

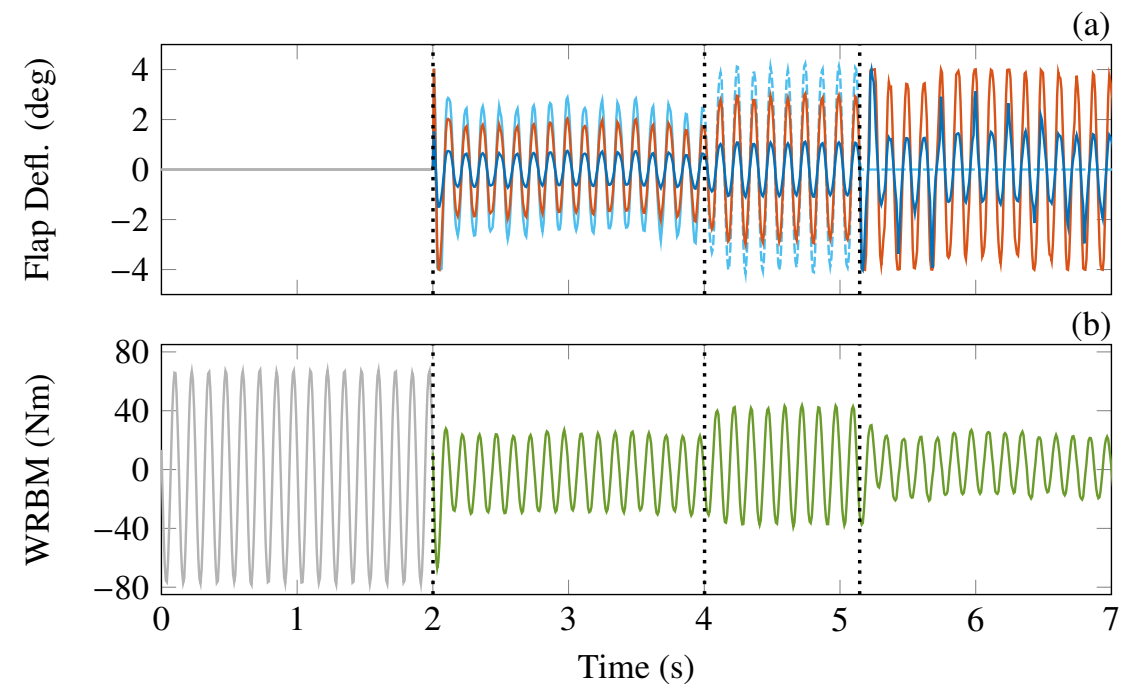

Fig. 12 Input and output data of the fault-tolerant control experiment with an outer flap actuator fault during sinusoidal pitch excitation. The commands for the inner $(-)$, mid $(-)$, and outer $(-)$ flap are depicted in (a), where (----) denotes that the outer flap command is not realized due to the simulated actuator fault. In (b), the measured wing root bending moment is depicted with open- and closed-loop operation indicated by $(-)$ and (-), respectively.

the pitch motor is present and excites the wing while the GLA controller is switched off. After around $2 \mathrm{~s}$ the GLA controller is switched on. Fig. 12(a) shows the deflections commanded to the inner (-), mid (-), and outer (-) flap counteracting the wing oscillations. In Fig. 12(b), the measured WRBM is depicted. As soon as the aeroelastic mode controller is activated, the WRBM is reduced by almost $60 \%$. At around $4 \mathrm{~s}$ the outer flap actuator is frozen at $2^{\circ}$. As the fault is present but the controller has not been reconfigured, commands to the outer flap are still present but not realized in the third part of the experiment, which is indicated by the dashed line (----). Due to the frozen actuator position, the WRBM increases as seen in Fig. 12(b). Compared to the open-loop case, however, the WRBM is still reduced since the mid and inner flaps are still in operation. After a fault detection time of a bit more than $1 \mathrm{~s}$, which is reasonable as evaluated in [15], the GLA controller is reconfigured and dynamic control allocation is activated at around $5 \mathrm{~s}$. From thereon, the demanded control effort is distributed to the two inner flaps while the command to the faulty actuator is set to zero. Clearly visible in Fig. 12(a) are the increased deflections commanded to the inner $(-)$ and mid (-) flap in the fourth segment. Thereby, controller performance in terms of WRBM reduction is not only recovered but actually outperformed as it can be seen in Fig. 12(b). The reason for this continues to be the large backlash of the inner flap as confirmed by nonlinear simulations. Furthermore, the peaks in the inner flap command are a compensation of the saturation of the mid flap command, which is limited to $\pm 4^{\circ}$ in the dynamic control allocation. This shows that the dynamic control allocation allows handling both deflection limitations and actuator faults at the same time. Within this final experiment, also the transition behavior of the GLA controller is investigated. As desired and visible in Fig. 12, the transitions between the different controller configurations are smooth and bumpless during the entire experiment. Based on the discussed results, the designed GLA system is successfully validated, demonstrating that the proposed combination of $\mathcal{H}_{2}$-optimal blending and dynamic control allocation is well suited for modal damping under actuator constraints.

\section{Conclusions}

In this paper, an advanced GLA control system is developed, and validated in an extensive wind tunnel test campaign. The used control approach is a combination of a gain-scheduled aeroelastic mode controller with a dynamic control allocation system. The aeroelastic mode controller is based on blending control inputs and measurement outputs, which not only reduces the complexity of the control design problem but also allows for a straight forward integration of the dynamic control allocation. It is shown that the proposed GLA system is capable dealing with actuator limitations like saturation or faults by redistributing control inputs in real-time. The encouraging results of the wind tunnel experiment pave the way for further research activities to achieve the goal of an in-flight validation of the algorithms in the future. 


\section{Acknowledgments}

The authors like to thank DLR's Institute of Aeroelasticity in Göttingen as well as the Institute of System Dynamics and Control in Oberpfaffenhofen for supporting the extensive wind tunnel test campaign. Special thanks goes out to Wolf Kürger, Marc Böswald, Holger Mai, Gertjan Looye, Jannis Lübker, Tobias Meier, Yasser Meddaikar and Ramesh Konatala for their valuable assistance. This work was conducted within the DLR-internal Aeronautical Research Project "KonTeKst".

\section{References}

[1] International Energy Agency, “Transport, Energy and $\mathrm{CO}_{2}$, , 2009.

[2] Regan, C., and Jutte, C., "Survey of Applications of Active Control Technology for Gust Alleviation and New Challenges for Lighter-weight Aircraft," Tech. Rep. NASA/TM-2012-216008, NASA, 2012.

[3] Fezans, N., Joos, H.-D., and Deiler, C., "Gust load alleviation for a long-range aircraft with and without anticipation," $C E A S$ Aeronautical Journal, Vol. 10, No. 4, 2019, pp. 1033-1057.

[4] Hecker, S., and Hahn, K.-U., “Advanced gust load alleviation system for large flexible aircraft," Proc. 1st CEAS Konferenz, Berlin, Germany, 2007.

[5] Pusch, M., Knoblach, A., and Kier, T., "Integrated optimization of control surface layout for gust load alleviation," CEAS Aeronautical Journal, 2019.

[6] Brockhaus, R., Alles, W., and Luckner, R., Flugregelung, Springer-Verlag, 2011.

[7] Ossmann, D., and Poussot-Vassal, C., "Minimal order disturbance estimator design for aircraft load alleviation control," 2018 IEEE Conference on Control Technology and Applications (CCTA), IEEE, 2018, pp. 787-793.

[8] Haghighat, S., Liu, H., and Martins, J. R., "Application of model predictive control to gust loads alleviation systems," AIAA Atmospheric Flight Mechanics Conference, 2009, p. 5929.

[9] Giesseler, H.-G., Kopf, M., Varutti, P., Faulwasser, T., and Findeisen, R., "Model predictive control for gust load alleviation," IFAC Proceedings Volumes, Vol. 45, No. 17, 2012, pp. 27-32.

[10] Kopf, M., Bullinger, E., Giesseler, H.-G., Adden, S., and Findeisen, R., "Model predictive control for aircraft load alleviation: Opportunities and challenges," 2018 Annual American Control Conference (ACC), IEEE, 2018, pp. 2417-2424.

[11] Kier, T., Leitner, M., Süelözgen, Ö., and Pusch, M., "An Integrated Flexible Aircraft Model for Optimization of Lift Distributions," AIAA Scitech 2019 Forum, San Diego, CA, USA, 2019.

[12] Johansen, T., and Fossen, T., “Control allocation - A Survey,” Automatica, Vol. 49, No. 5, 2013, pp. 1087-1103.

[13] Frost, S., Taylor, B., and Bodson, M., "Investigation of optimal control allocation for gust load alleviation in flight control," AIAA atmospheric flight mechanics conference, 2012, p. 4858.

[14] Hansen, J. H., Duan, M., Kolmanovsky, I., and Cesnik, C. E., "Control allocation for maneuver and gust load alleviation of flexible aircraft," AIAA Scitech 2020 Forum, 2020, p. 1186.

[15] Ossmann, D., and Pusch, M., "Fault Tolerant Control of an Experimental Flexible Wing," Aerospace, Vol. 6, No. 7, 2019.

[16] Jain, T., and Yamé, J. J., "Fault-tolerant economic model predictive control for wind turbines," IEEE transactions on sustainable energy, Vol. 10, No. 4, 2018, pp. 1696-1704.

[17] Pusch, M., and Ossmann, D., “'H 2 -optimal Blending of Inputs and Outputs for Modal Control," IEEE Transaction of Control System Technology, 2019.

[18] Pusch, M., and Ossmann, D., "Blending of Inputs and Outputs for Modal Velocity Feedback," 27th IEEE Mediterranean Conference on Control and Automation, Akko, Israel, 2019, pp. 1-10.

[19] Pusch, M., Theis, J., and Ossmann, D., "Partial Pole Placement using Static Output Feedback," IFAC-PapersOnLine, Vol. 53, 2020, pp. 4527-4533.

[20] Baár, T., and Luspay, T., “An $\mathcal{H}_{2} / \mathcal{H}_{\infty}$ blending for mode decoupling,” 2019 American Control Conference, 2019 , pp. 175-180. 
[21] Baár, T., Bauer, P., and Luspay, T., "Parameter Varying Mode Decoupling for LPV systems," IFAC-PapersOnLine, Vol. 53, 2020, pp. 1219-1224.

[22] Baár, T., and Luspay, T., "Decoupling through input-output blending," International Journal of Control, 2020, pp. 1-15.

[23] Pusch, M., "Blending of inputs and outputs for modal control of aeroelastic systems," Ph.D. thesis, Technical Univesity Hamburg, 2020.

[24] Murty, K., Linear programming, John Wiley \& Sons, 1983.

[25] Boyd, S., and Vandenberghe, L., Convex optimization, Cambridge university press, 2004.

[26] Bodson, M., "Evaluation of Optimization Methods for Control Allocation," Journal of Guidance, Control, and Dynamics, Vol. 25, No. 4, 2002, pp. 703-711.

[27] Rugh, W. J., and Shamma, J. S., "Research on gain scheduling,” Automatica, Vol. 36, No. 10, 2000, pp. 1401-1425.

[28] Wu, F., “Control of linear parameter varying systems," Ph.D. thesis, University of California, Berkeley, 1995.

[29] Balas, G., "Linear, parameter-varying control and its application to aerospace systems," ICAS congress proceedings, 2002.

[30] Theis, J., Robust and linear parameter-varying control of aeroservoelastic systems, Technische Universität Hamburg-Harburg, 2018.

[31] Hoffmann, C., Linear parameter-varying control of systems of high complexity, Technische Universität Hamburg, 2016.

[32] Apkarian, P., Gahinet, P., and Buhr, C., "Multi-model, multi-objective tuning of fixed-structure controllers," European Control Conference, IEEE, 2014, pp. 856-861.

[33] Apkarian, P., Dao, M. N., and Noll, D., "Parametric Robust Structured Control Design," IEEE Transactions on Automatic Control, Vol. 60, No. 7, 2015, pp. 1857-1869. https://doi.org/10.1109/TAC.2015.2396644.

[34] Pusch, M., Ossmann, D., and Luspay, T., "Structured Control Design for a Highly Flexible Flutter Demonstrator," Aerospace, Vol. 6, No. 3, 2019.

[35] Krüger, W. R., Dillinger, J., Meddaikar, M. Y., Lübker, J., Tang, M., Meier, T., Pusch, M., and Kier, T., "Design and Wind Tunnel Test of an Actively Controlled Flexible Wing," International Forum on Aeroelasticity and Structural Dynamics, Savannah, GA, USA, 2019, pp. 1-15.

[36] Pusch, M., Ossmann, D., Dillinger, J., Kier, T., Tang, M., and Lübker, J., "Aeroelastic Modeling and Control of an Experimental Flexible Wing," AIAA Guidance, Navigation, and Control Conference, San Diego, USA, 2019, pp. 1-15.

[37] Dillinger, J. K. S., Meddaikar, Y. M., Lübker, J., Pusch, M., and Kier, T., "Design and Optimization of an Aeroservoelastic Wind Tunnel Model,” Fluids, Vol. 5, No. 1, 2020.

[38] Tang, M., Böswald, M., Govers, Y., and Pusch, M., "Identification and assessment of a nonlinear dynamic actuator model for gust load alleviation in a wind tunnel experiment," Deutscher Luft- und Raumfahrtkongress (DLRK), Darmstadt, Germany, 2019, pp. 1-10.

[39] Pusch, M., "Aeroelastic Mode Control using $\mathcal{H}_{2}$-optimal Blends for Inputs and Outputs," AIAA Guidance, Navigation, and Control Conference, Orlando, FL, USA, 2018.

[40] Blight, J., Dailey, L., and Gangsaas, D., "Practical control law design for aircraft using multivariable techniques," International Journal of Control, Vol. 59, No. 1, 1994, pp. 93-137.

[41] Schmid, C., and Biegler, L., "Quadratic programming methods for reduced Hessian SQP," Computers \& chemical engineering, Vol. 18, No. 9, 1994, pp. 817-832.

[42] Hoblit, F., Gust loads on aircraft: concepts and applications, AIAA, 1988. 